\title{
On the Distribution of the Sum of Generalized Gamma Variates and Applications to Satellite Digital Communications
}

\author{
Nikos C. Sagias, P. Takis Mathiopoulos, and Petros S. Bithas \\ Institute for Space Applications and Remote Sensing, \\ National Observatory of Athens. \\ Metaxa \& Vas. Pavlou Street, 15236 Athens, Greece \\ E-mail: \{nsagias;mathio;bithas\}@space.noa.gr
}

\author{
George K. Karagiannidis \\ Division of Telecommunications, \\ Department of Electrical and Computer Engineering, \\ Aristotle University of Thessaloniki, 54124 Thessaloniki, Greece \\ E-mail: geokarag@auth.gr
}

\begin{abstract}
A versatile envelope distribution which may be useful for both terrestrial and satellite fading channel modelling is the generalized Gamma (GG) distribution. By considering the product of $N$ GG random variables (RV)s, useful expressions for its moments-generating and cumulative distribution functions are obtained in closed-form. These expressions are used to derive a closed-form union upper-bound for the distribution of the sum of GG distributed RVs. The proposed bound turns out to be an extremely convenient analytical tool for studying the performance of $N$-branch equal-gain combining receivers operating over GG fading channels. For such receivers, novel union upper-bounds for the outage and the average bit error probability are derived and evaluated in terms of the Meijer's G-function. The tightness of the proposed bounds is verified by performing comparisons between numerical evaluation and computer simulations results.
\end{abstract}

\section{INTRODUCTION}

A general envelope distribution which includes many wellknown channel models for both multipath as well as for shadow fading is the so-called generalized Gamma (GG) distribution. This distribution was introduced by Stacy, back in 1962, as a generalization of the (two-parameter) Gamma distribution [1] and it includes the Rayleigh, Nakagami- $m$, Weibull, and Lognormal. Due its great flexibility, it may be used in statistical modelling of both terrestrial and satellite wireless applications for which only very recently the topic of performance analysis of digital receivers over this generalized channel has gained renewed interest. Particularly, in an early work on this topic [2], Coulson et al. presented expressions for the average bit error probability (ABEP) of single-branch receivers operating over a GG fading environment, with binary phase-shift keying (BPSK) and binary frequency-shift keying (BFSK) modulations, as infinite series. In another related work [3], Yacoub introduced the $\alpha-\mu$ distribution and gave a physical justification for the origin of the GG model. More recently, Aalo et al. presented a closed-form expression for the ABEP for both coherent and noncoherent/differentially coherent binary digital modulations [4].

The performance of diversity receivers has been extensively studied in the past for the most important fading channel models, Rayleigh, Nakagami- $m$, and Weibull (e.g. see [5]-
[8]). However, a performance study of diversity and specifically equal-gain combining (EGC) receivers over GG fading channels has not been presented yet. The main difficulty in studying EGC receivers is that the distribution of the sum of fading envelopes is required. The derivation of this distribution in terms of tabulated functions is a very difficult task [5]. Concerning this well-recognized but cumbersome statistical problem, several approaches aiming in providing possible solutions have been published in the open technical literature. In possibly one of the earliest works, Stacy in his original GG paper developed an infinite series approach for determining the cumulative density function (cdf) of the sum of GG distributed random variables (RV)s [1]. Many years later, in an independent from [1] approach, Beaulieu derived an infinite series approach for determining the cdf of the sum of Rayleigh distributed RVs [9]. Helstrom has computed the distribution of such a sum using saddle-point integration for uniformly weighted RVs [10], as well as for arbitrary weights [11]. Filho and Yacoub in [12] have derived an approximated probability density function (pdf) expression for the sum of Nakagami-m RVs. Very recently, Karagiannidis et al. have presented a closed-form union upper-bound for the cdf of the weighted sum of $N$ independent Rayleigh RVs [13]. All in all, although the problem of finding the distribution of the sum of fading envelopes has been extensively studied in the past for various distributions, the majority of the published methods are approximate solutions usually involving a truncation error. Hence, the derivation of an exact solution, in terms of tabulated functions, even for the simplest Rayleigh distribution, when $N>2$ and with nonidentical statistical parameters, still remains an open research problem.

In this paper, in an effort to provide a solution to this problem and within the framework of studying the performance of EGC receivers over GG fading channels, another approach is proposed. Since an analytical solution for the distribution of the sum of RVs is very difficult to be derived, the use of union bounds is proposed. In particular, by deriving a useful expression for the cdf of the product of $N$ GG RVs and based on the well-known inequality between arithmetic and geometric means, closed-form union upper-bounds for the cdf 
of the sum of GG distributed RVs are obtained. These bounds, which turn out to be quite tight, are used to analyze the ABEP and outage performance of $N$-branch EGC receivers operating over GG fading channels.

\section{The GG Distribution and Its Product}

Let us consider $N \geq 1$ independent three-parameters' GG distributed RVs $\left\{R_{\ell}\right\}_{\ell=1}^{N}$ with pdf given by [1, eq. (1)]

$$
f_{R_{\ell}}(r)=\frac{\beta_{\ell} r^{m_{\ell} \beta_{\ell}-1}}{\left(\Omega_{\ell} / m_{\ell}\right)^{m_{\ell}} \Gamma\left(m_{\ell}\right)} \exp \left(-\frac{m_{\ell}}{\Omega_{\ell}} r^{\beta_{\ell}}\right)
$$

where $\beta_{\ell}>0$ and $m_{\ell} \geq 1 / 2$ are two parameters related to the fading severity, $\Omega_{\ell}$ is related to the average fading power as $\mathcal{E}\left\langle R_{\ell}^{2}\right\rangle=\left(\Omega_{\ell} / m_{\ell}\right)^{2 / \beta_{\ell}} \Gamma\left(m_{\ell}+2 / \beta_{\ell}\right) / \Gamma\left(m_{\ell}\right)$, with $\mathcal{E}\langle\cdot\rangle$ denoting expectation, and $\Gamma(\cdot)$ being the Gamma function [14, eq. (8.310/1)]. The distribution in (1) is very generic ${ }^{1}$ since it includes commonly used fading models such as Rayleigh (for $\beta_{\ell}=2$ and $m_{\ell}=1$ ), Nakagami-m (for $\beta_{\ell}=2$ ), and Weibull (for $m_{\ell}=1$ ) as special cases. Moreover, for the limiting case of $\beta_{\ell} \rightarrow 0$ and $m_{\ell} \rightarrow \infty$, (1) becomes the well-known Lognormal pdf. The the $n$th order moment of $R_{\ell}$ can be expressed as

$$
\mathcal{E}\left\langle R_{\ell}^{n}\right\rangle=\left(\frac{\Omega_{\ell}}{m_{\ell}}\right)^{n / \beta_{\ell}} \frac{\Gamma\left(m_{\ell}+n / \beta_{\ell}\right)}{\Gamma\left(m_{\ell}\right)}
$$

where $n$ is a positive number.

\section{A. Distribution of the Product of GG Variates}

Let us define another RV, $Y$, as the product of the $N$ GG distributed RVs $R_{\ell}$, i.e., $Y \triangleq \prod_{i=1}^{N} R_{i}$.

Theorem 1: The moments-generating function (mgf) of $Y$ is given by

$$
\mathcal{M}_{Y}(s)=V G_{n, p}^{p, n}\left[\frac{s^{-n}}{W} \mid \begin{array}{c}
\Delta(n ; 1) \\
\Delta\left(\frac{n}{\beta_{1}} ; m_{1}\right), \Delta\left(\frac{n}{\beta_{2}} ; m_{2}\right), \ldots, \Delta\left(\frac{n}{\beta_{N}} ; m_{N}\right)
\end{array}\right]
$$

where $G[\cdot]$ is the Meijer's G-function [14, eq. (9.301)] and $\Delta(k ; x)$ is defined as $\Delta(k ; x) \triangleq x / k,(x+1) / k, \ldots,(x+$ $k-1) / k$, with $x$ being an arbitrary real value and $k$ a positive integer,

$$
V=\sqrt{n}(\sqrt{2 \pi})^{N+1-n-p} \prod_{i=1}^{N} \frac{\left(n / \beta_{i}\right)^{m_{i}-1 / 2}}{\Gamma\left(m_{i}\right)}
$$

and

$$
W=\frac{1}{n^{n}} \prod_{i=1}^{N}\left(\frac{n \Omega_{i}}{m_{i} \beta_{i}}\right)^{n / \beta_{i}}
$$

Moreover, $n$ and $p$ are two positive integers defined as $n \triangleq$ $\prod_{i=1}^{N} k_{i}$ and $p \triangleq n \sum_{i=1}^{N} 1 / \beta_{i}$ under the constraint that $l_{\ell}=$ $\prod_{i=1}^{\ell} k_{i} / \beta_{\ell}$ is a positive integer, with $k_{\ell}$ and $l_{\ell}$ being also positive integers.

\footnotetext{
${ }^{1}$ As pointed out in [4], the pdf of (1), introduced by Stacy in [1], is different from the type of generalization for the Gamma distribution presented in [15] which models the power of Rice-faded envelopes.
}

Proof: The proof is given in the full version of this paper $^{2}$.

The values for $k_{\ell}$ and $l_{\ell}$ can be found as follows: Depending upon the value $\beta_{1}, k_{1}$ and $l_{1}$ are two minimum positive integers such that $l_{1}=k_{1} / \beta_{1}$ holds (e.g. for $\beta_{1}=3.6, k_{1}=18$ and $l_{1}=5$ ). Similarly, $k_{\ell}$ and $l_{\ell}$ are two minimum positive integers such that $l_{\ell}=\prod_{i=1}^{\ell} k_{i} / \beta_{\ell}$ holds. Note that for identical and integer order fading parameters (3) significantly simplifies.

Lemma 1 (Cumulative distribution function): The cdf of $Y$ is given by

$$
\begin{aligned}
& F_{Y}(y)=\frac{V}{\sqrt{n}}(\sqrt{2 \pi})^{n-1}
\end{aligned}
$$

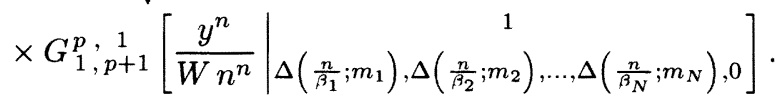

Proof: Applying the inverse Laplace transform $\mathcal{L}^{-1}(\cdot ; \cdot)$ [14, Sec. 17.11] in (3), the pdf of $Y\left[14\right.$, Sec. 17.11] $f_{Y}(y)=$ $\mathcal{L}^{-1}\left\{\mathcal{M}_{Y}(s) ; y\right\}$ can be obtained in closed-form using [16, eq. (21)]. Since the cdf of $Y$ is given by $F_{Y}(y)=\int_{0}^{y} f_{Y}(x) d x$ and by using [16, eq. (26)], (5) results.

\section{B. Distribution of the Sum of $G G$ Variates}

Let us define $S$ to be the sum of $N$ GG RVs, i.e., $S \triangleq$ $\sum_{i=1}^{N} R_{i}$.

Theorem 2: The cdf of $S$ is upper-bounded as

$$
F_{S}(y) \leq F_{Y}\left[\left(\frac{y}{N}\right)^{N}\right] .
$$

Proof: Using the well-known inequality for the arithmetic and geometric means [14, Sec. 11.116]

$$
\mathcal{A}_{N} \geq \mathcal{G}_{N}
$$

with $\mathcal{A}_{N} \triangleq \sum_{i=1}^{N} R_{i} / N$ and $\mathcal{G}_{N} \triangleq \prod_{i=1}^{N} R_{i}^{1 / N}$ being the arithmetic and geometric means, respectively, $S$ can be lowerbounded as $S \geq N \prod_{i=1}^{N} R_{i}^{1 / N}$. Using (5), it can be easily seen that the cdf of $S$ can be upper-bounded as in (6).

It is interesting to note that the problem of obtaining the cdf of $S$ with nonidentically distributed RVs $R_{\ell}$ may be equivalently stated as finding the weighted sum of $N$ i.i.d. RVs having $\Omega_{\ell}=\Omega$ average power each, with weights $w_{\ell}=\sqrt{\Omega_{\ell} / \Omega}$.

\section{Performance Analysis of Equal-Gain DIVERSITY RECEIVERS}

Let us consider an $N$-branch EGC receiver operating over independent, but not necessarily identically distributed, GG fading channels. The baseband received signal in the $\ell$ th $(\ell=$ $1,2, \ldots, N)$ antenna is $\zeta_{\ell}=s R_{\ell} \exp \left(\jmath \psi_{\ell}\right)+n_{\ell}$, where $s$ is the complex transmitted symbol, with $E_{s}=\mathcal{E}\left\langle|s|^{2}\right\rangle$ being the transmitted average symbols' energy, $R_{\ell}$ is the instantaneous fading envelope being modelled as a GG distributed RV, $\psi_{\ell}$ is the instantaneous phase of the channel, and $n_{\ell}$ is the

${ }^{2}$ N. C. Sagias, G. K. Karagiannidis, P. T. Mathiopoulos, and T. A. Tsiftsis, "On the performance analysis of equal-gain diversity receivers over generalized Gamma fading channels," sumbitted to the IEEE Trans. Wireless Commun. 


$$
\begin{aligned}
& F_{\gamma_{e g c}}(\gamma)=\frac{(\sqrt{2 \pi})^{n-1}}{\sqrt{n} / V} G_{1, p+1}^{p, 1}\left[\frac{(\gamma / N)^{n N}}{W n^{n}} \mid \Delta\left(\frac{2 n}{\beta_{1}} ; m_{1}\right), \Delta\left(\frac{2 n}{\beta_{2}} ; m_{2}\right), \ldots, \Delta\left(\frac{2 n}{\beta_{N}} ; m_{N}\right), 0\right] \\
& \bar{P}_{s e} \leq \frac{A V}{\sqrt{\pi n}} \frac{1}{(\sqrt{2 \pi})^{n(N-1)}} G_{2 n N, p+n N}^{p, 2 n N}\left[\left.\frac{n^{n(N-1)}}{W B^{n N}}\right|_{\Delta\left(2 n / \beta_{1} ; m_{1}\right), \Delta\left(2 n / \beta_{2} ; m_{2}\right), \ldots, \Delta\left(2 n / \beta_{N} ; m_{N}\right), \Delta(n N ; 0)}\right] \\
& \bar{P}_{s e} \leq \frac{A V \sqrt{N}}{(\sqrt{2 \pi})^{n(N-1)}} G_{n N, p}^{p, n N}\left[\left.\frac{n^{n(N-1)}}{W B^{n N}}\right|_{\Delta\left(2 n / \beta_{1} ; m_{1}\right), \Delta\left(2 n / \beta_{2} ; m_{2}\right), \ldots, \Delta\left(2 n / \beta_{N} ; m_{N}\right)}\right]
\end{aligned}
$$

instantaneous additive white Gaussian noise (AWGN) sample with single-sided power spectral density $N_{0}$ identical to all channels. The usual assumption is made that the $\psi_{\ell}$ 's are known to the receiver.

The instantaneous SNR per symbol at the $\ell$ th diversity input channel, $\gamma_{\ell}$, is $\gamma_{\ell}=R_{\ell}^{2} E_{s} / N_{0}$ with its corresponding average SNR being $\bar{\gamma}_{\ell}=\left(m_{\ell}\right)_{2 / \beta_{\ell}}\left(\Omega_{\ell} / m_{\ell}\right)^{2 / \beta_{\ell}} E_{s} / N_{0}$ where $(\xi)_{u}$ is the Pochhammer symbol defined as $(\xi)_{u}=\Gamma(\xi+u) / \Gamma(\xi)$. Based on an interesting property of the GG distribution, that the $n$th power of a GG distributed RV with parameters $\left(m_{\ell}, \beta_{\ell}, \Omega\right)$ is another GG distributed RV with parameters $\left(m_{\ell}, \beta_{\ell} / n, \Omega\right)$, it can be easily concluded that $\gamma_{\ell}$ is also a GG distributed RV with parameters $\left(m_{\ell}, \beta_{\ell} / 2,\left(\Xi_{\ell} \bar{\gamma}_{\ell}\right)^{\beta_{\ell} / 2}\right)$ with $\Xi_{\ell}=1 /\left(m_{\ell}\right)_{2 / \beta_{\ell}}$. Hence, by using the formulae for $\left\{R_{\ell}\right\}$ presented in the previous section, the corresponding expressions for $\left\{\gamma_{\ell}\right\}$ can be easily derived, replacing $\beta_{\ell}$ with $\beta_{\ell} / 2$ and $\Omega_{\ell} / m_{\ell}$ with $\left(\Xi_{\ell} \bar{\gamma}_{\ell}\right)^{\beta_{\ell} / 2}$ helping us to study the performance of multi-branch diversity receivers operating over GG fading channels.

\section{A. Outage Probability}

The cdf of $\gamma_{e g c}$ can be derived by substituting $y=(\gamma / N)^{N}$ in (5), i.e., as in (8) (see at top of this page).

If $\gamma_{t h}$ is a certain specified threshold, then the outage probability is defined as the probability that the EGC output SNR falls below $\gamma_{t h}$. An upper-bound for this probability can be obtained by replacing $\gamma$ with $\gamma_{t h}$ in (8) as

$$
P_{\text {out }}\left(\gamma_{t h}\right) \leq F_{\gamma_{e g c}}\left(\gamma_{t h}\right)
$$

\section{B. Average Bit Error Probability}

The most straightforward approach to obtain the ABEP, $\bar{P}_{b e}$, is to average the conditional symbol error probability $P_{b e}(\gamma)$ over the pdf of $\gamma$, i.e., $\bar{P}_{b e}=\int_{0}^{\infty} P_{b e}(\gamma) f_{\gamma_{e g c}}(\gamma) d \gamma$. By taking the first derivative of (8) with respect to $\gamma$, the corresponding pdf can be obtained as

$$
\begin{aligned}
& f_{\gamma_{e g c}}(\gamma)=\frac{N \sqrt{n} V(\sqrt{2 \pi})^{n-1}}{\gamma} \\
& \times G_{0, p}^{p, 0}\left[\left.\frac{(\gamma / N)^{n N}}{W n^{n}}\right|_{\Delta\left(\frac{2 n}{\beta_{1}} ; m_{1}\right), \Delta\left(\frac{2 n}{\beta_{2}} ; m_{2}\right), \ldots, \Delta\left(\frac{2 n}{\beta_{N}} ; m_{N}\right)} ^{-} .\right.
\end{aligned}
$$

Moreover, for $P_{b e}(\gamma)$ there are well-known generic expressions for two different sets of modulation schemes: i) For BPSK, BFSK, $M$-ary differentially encoded phaseshift keying ( $M$-DEPSK), quadrature phase-shift keying (QPSK), $M$-ary phase-shift keying ( $M$-PSK), $M$ ary frequency-shift keying ( $M$-FSK), square $M$-ary quadrature amplitude modulation ( $M$-QAM), and $M$ ary differential PSK ( $M$-DPSK) in the form of $P_{b e}(\gamma)=$ $A \operatorname{erfc}(\sqrt{B \gamma})$ where $\operatorname{erfc}(\cdot)$ is the well-known complementary error function [14, eq. (8.250/4)];

ii) For differential BPSK (DBPSK) and $M$-ary noncoherent frequency-shift keying ( $M$-NFSK), in the form $P_{b e}(\gamma)=A \exp (-B \gamma)$.

The particular values of $A$ and $B$ depend on the specific modulation scheme employed and can be found in [5]. Next, $\bar{P}_{b e}$ is found in closed-form for the above two sets of signals.

1) BFSK, $M$-DEPSK, $M$-PSK, $M$-FSK, $M$-QAM, and $M$ $D P S K$ : Using (10) and in order to derive $\bar{P}_{b e}$ for this first set of modulated schemes, it can be easily recognized that the evaluation of definite integrals, which include Meijer's, power, and exponential functions, is required. Since such integrals are not tabulated, the solution can be found with the aid of [16, eq. (21)], and thus the ABEP can be upper-bounded as in (13) (see at top of this page).

2) DBPSK and M-NFSK: Similarly to the first set, for this second set (i.e., for NBFSK and DBPSK), the ABEP can be derived as in (14) (see at top of this page).

\section{Performance Evaluation and Discussion}

In this section, using the previous mathematical analysis, numerical and simulation results are presented for the performance of EGC receivers operating over GG fading channels. For these performance evaluation results we consider the general case of not necessarily equal $\bar{\gamma}_{\ell}$ 's. Particularly, an exponentially decaying power delay profile (PDP) is adopted $\bar{\gamma}_{\ell}=\bar{\gamma}_{1} \exp [-\delta(\ell-1)]$ with $\delta$ being the power decaying factor. For the convenience of the presentation of the performance evaluation results and without any loss of generality, it will be assumed that $m_{\ell}=m$ and $\beta_{\ell}=\beta \forall \ell$.

Having numerically evaluated (9), in Fig. 1, upper-bounds for $P_{\text {out }}$ are presented as a function of the normalized outage threshold, $\gamma_{t h} / \bar{\gamma}$, for $m=2$, i.i.d. input branches (i.e., $\bar{\gamma}_{\ell}=\bar{\gamma}$ ), and different values of $\beta$ and $N$. The obtained results clearly show that $P_{\text {out }}$ improves with an increase of $N$ and/or $\beta$. In order to verify the tightness of the bounds, curves obtained by means of computer simulations are also included for comparison purposes. By comparing the performances 


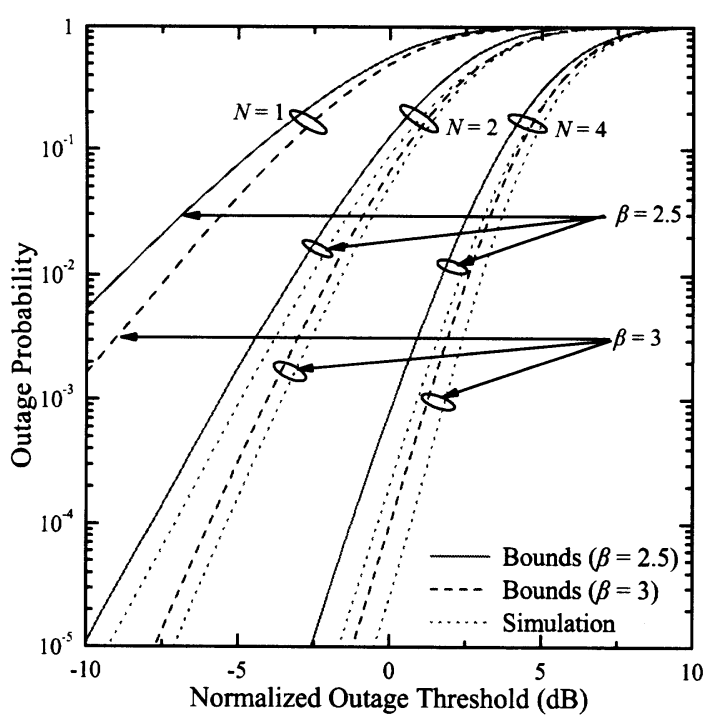

Fig. 1. Outage probability $\left(P_{\text {out }}\right)$ as a function of the average input SNR for $m=2$ and i.i.d. branch SNRs.

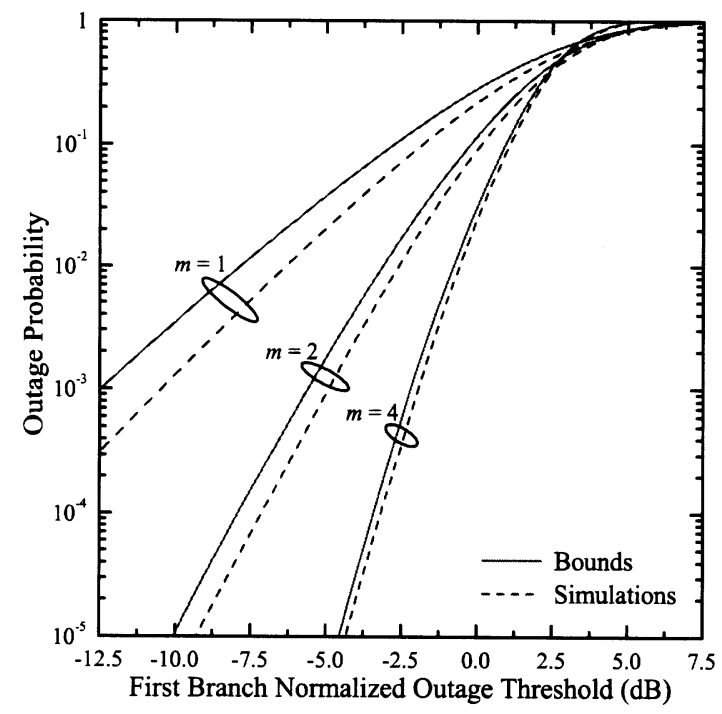

Fig. 2. Outage probability as a function of the first branch normalized average input SNR for $\beta=2.5$ and $\bar{\gamma}_{1}=0.5 \bar{\gamma}_{2}$.

it is evident that the numerical results for the bounds (see (9)) are very close to the equivalent simulated ones which represent the exact $P_{\text {out }}$ performance. This observation clearly demonstrates the accuracy of the proposed bounds. It is also noted that as $\beta$ increases, the proposed bounds become even tighter. However, as $N$ increases, the difference between the two performance results slightly increases. In Fig. $2, P_{\text {out }}$ is plotted as a function of the first branch normalized outage threshold, $\gamma_{t h} / \bar{\gamma}_{1}$, for $\beta=2.5, N=2$, and $\bar{\gamma}_{1}=0.5 \bar{\gamma}_{2}$. These results suggest that the higher $m$ is, the smaller are the differences between numerical and computer simulation results for $P_{\text {out }}$. For example, at $P_{\text {out }}=10^{-3}$, the differences between them for $m=1,2$, and 4 are less than 2,1 , and 0.5

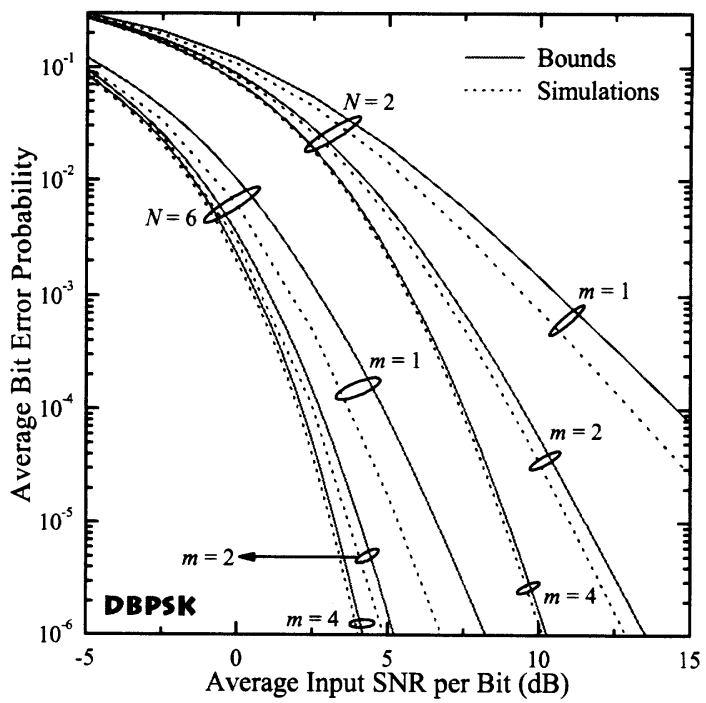

Fig. 3. ABEP of EGC with DBPSK modulation format as a function of the average input SNR per bit for $\beta=3$ and i.i.d. branch SNRs.

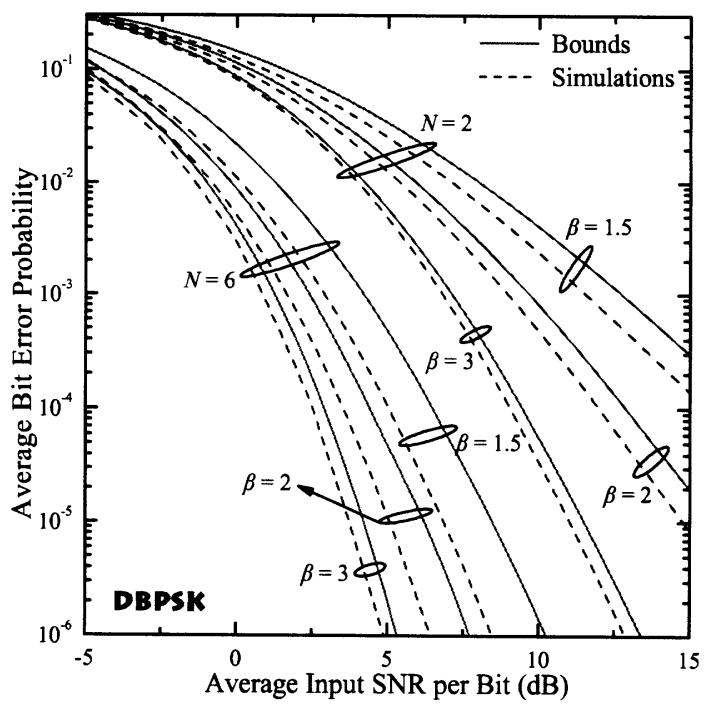

Fig. 4. ABEP of EGC with DBPSK modulation format as a function of the average input SNR per bit for $m=2$ and i.i.d. branch SNRs.

$\mathrm{dB}$, respectively. The trend of the performance, as illustrated in Figs. 1 and 2, can be explained as follows. It is clear that the lower the difference between the terms of the left hand side (LHS) and right hand side (RHS) of (7), the tighter are the bounds. In fact, the equality in (7) holds if and only if all $R_{\ell}$ 's are equal with each other, i.e., $R_{1}=R_{2}=\cdots=R_{N}$. For relatively large values of $m_{\ell}$ 's and/or $\beta_{\ell}$ 's, all fading envelopes $R_{\ell}$ will be, with high probability, close to their average value, and thus, it is expected that $R_{\ell}$ 's will take similar values. As for $N$, the lower its value is the tighter are the bounds. This happens because both bounds and exact curves move towards the performance obtained for $N=1$. In fact, from (7) it can be seen that for $N=1$ these two curves coincide. 


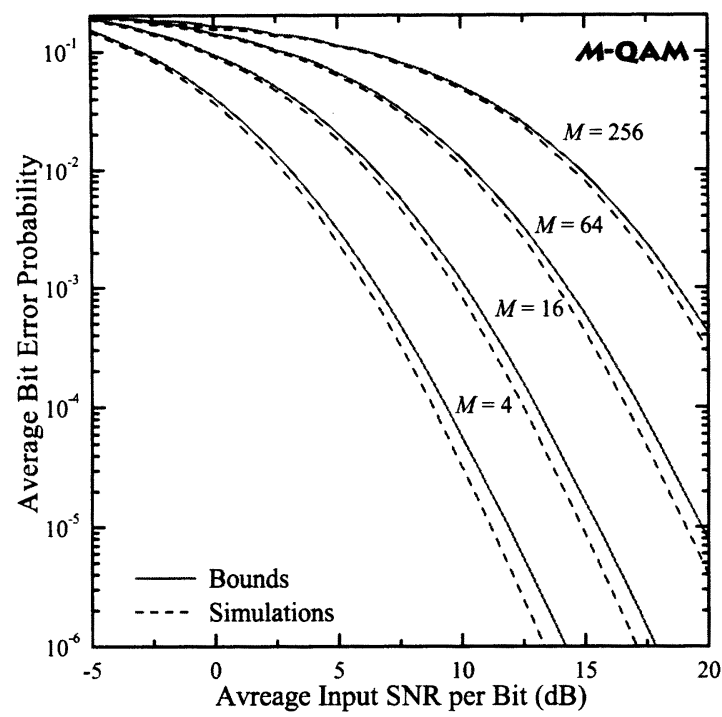

Fig. 5. ABEP of EGC with Gray encoded $M$-QAM modulation format as a function of the average input SNR per bit for $m=2 \beta=2.5$ and i.i.d. branch SNRs.

Using (13) and (14), the ABEP, $\bar{P}_{b e}$, of $N$-branch EGC receivers for several coherent and noncoherent binary and multilevel modulation schemes can be obtained. In Figs. 3 and $4, \bar{P}_{b e}$ of DBPSK is plotted as a function of $\bar{\gamma}$ for i.i.d. fading statistics. In Fig. 3, $\bar{P}_{b e}$ is plotted as a function of $\bar{\gamma}$, for $\beta=3$ and several values of $m$. As expected, the obtained performance evaluation results show that $\bar{P}_{b e}$ improves with an increase of $\bar{\gamma}$. For comparison purposes the curves for the corresponding exact $\bar{P}_{b e}$, obtained via computer simulations, are also included in the same figure. By comparing the numerically evaluated results with the computer simulated ones, we deduce a close match between them. In Fig. $4, \bar{P}_{b e}$ is plotted as a function of $\bar{\gamma}$, for $m=2$ and several values of $\beta$. As for $m, \beta$, and $N$, similar findings with those observed from Figs. 1 and 2 can also extracted. Fig. 5 presents $\bar{P}_{b e}$ of $M$-QAM with Gray encoding as a function of $\bar{\gamma}$ for $m=2$, $\beta=2.5$, i.i.d. input branches, and several values of $M$. As expected, for a fixed $\bar{\gamma}, \bar{P}_{b e}$ degrades with increasing $M$. Furthermore, the higher $M$, the tighter the bounds. Finally, in Fig. 6, $\bar{P}_{b e}$ of Gray coded 8-PSK is plotted as a function of $\bar{\gamma}_{1}$ for $\beta=2.5, \delta=0.2$, and $M=8$. Again here, note the tightness of the proposed bounds.

\section{ACKNOWLEDGMENT}

This work has been performed within the framework of the Satellite Network of Excellence (SatNEx) project, a Network of Excellence (NoE) funded by European Committee (EC) under the FP6 program.

\section{REFERENCES}

[1] E. W. Stacy, "A generalization of the Gamma distribution," Ann. Math. Stat., vol. 33, no. 3, pp. 1187-1192, Sept. 1962.

[2] A. J. Coulson, A. G. Williamson, and R. G. Vaughan, "Improved fading distribution for mobile radio," IEE Proc. Commun., vol. 145, no. 3, pp. 197-202, June 1998.

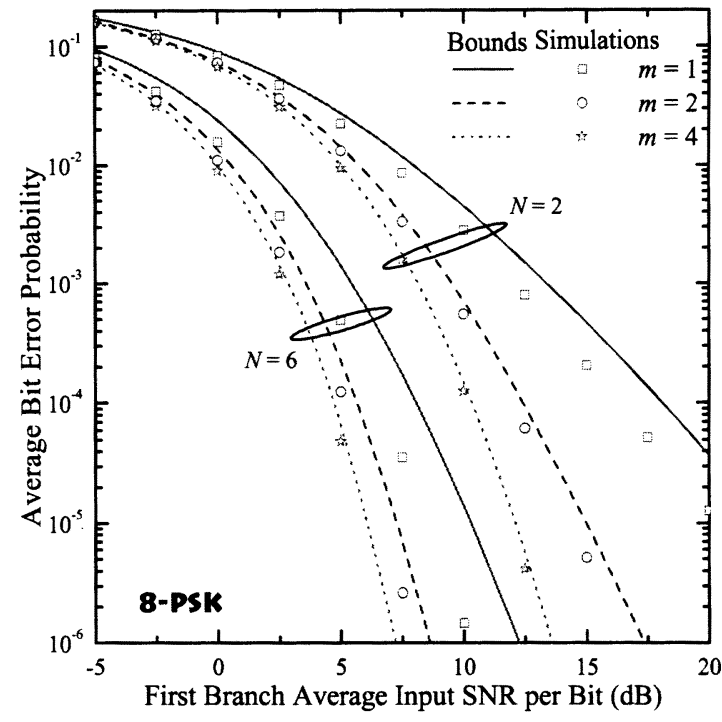

Fig. 6. ABEP of EGC with Gray encoded 8-PSK modulation format as a function of the average input SNR per bit for $\beta=2.5$ and $\delta=0.2$.

[3] M. D. Yacoub, "The $\alpha-\mu$ distribution: A general fading distribution," in Proc. IEEE International Symposium on Personal, Indoor, Mobile Radio Communications, vol. 2, Lisbon, Sept. 2002, pp. 629-633.

[4] V. A. Aalo, T. Piboongungon, and C.-D. Iskander, "Bit-error rate of binary digital modulation schemes in generalized Gamma fading channels," IEEE Commun. Lett., vol. 9, no. 2, pp. 139-141, Feb. 2005.

[5] M. K. Simon and M.-S. Alouini, Digital Communication over Fading Channels, 2nd ed. New York: Wiley, 2004.

[6] N. C. Beaulieu and A. Abu-Dayya, "Analysis of equal gain diversity on Nakagami fading channels," IEEE Trans. Commun., vol. 39, no. 2, pp. 225-234, Feb. 1991

[7] J. Cheng, C. Tellambura, and N. C. Beaulieu, "Performance of digital linear modulations on Weibull slow-fading channels," IEEE Trans. Commun., vol. 52, no. 8, pp. 1265-1268, Aug. 2004.

[8] G. K. Karagiannidis, D. A. Zogas, N. C. Sagias, S. A. Kotsopoulos, and G. S. Tombras, "Equal-gain and maximal-ratio combining over nonidentical Weibull fading channels," IEEE Trans. Wireless Commun., vol. 4 , no. 3, May 2005.

[9] N. C. Beaulieu, "An infinite series for the computation of the complementary probability distribution function of a sum of independent random variables and its application to the sum of Rayleigh random variables," IEEE Trans. Commun., vol. 38, no. 10, pp. 1463-1474, Sept. 1990.

[10] C. W. Helstrom, "Performance of receivers with linear detectors," IEEE Trans. Aerosp. Electron. Syst., vol. 26, pp. 210-217, Mar. 1990.

[11] _ "Computing the distribution of sums of random sine waves and of Rayleigh-distributed random variables by saddle-point integration," IEEE Trans. Commun., vol. 45, no. 11, pp. 1487-1494, Nov. 1997.

[12] J. C. S. S. Filho and M. D. Yacoub, "Nakagami- $m$ approximation to the sum of $m$ non-identical independent Nakagami- $m$ variates," Electron. Lett., vol. 40, no. 15, pp. 951-952, July 2004.

[13] G. K. Karagiannidis, T. A. Tsiftsis, and N. C. Sagias, "A closedform upper-bound for the distribution of the weighted sum of Rayleigh variates," IEEE Commun. Lett., to apperar in 2005.

[14] I. S. Gradshteyn and I. M. Ryzhik, Table of Integrals, Series, and Products, 6th ed. New York: Academic, 2000.

[15] J. Cheng and T. Berger, "Performance analysis for MRC and postdetection EGC over generalized Gamma fading channels," in Proc. IEEE Wireless Commun. and Networking Conference, vol. 1, New Orleans, LA, Mar. 2003, pp. 120-125.

[16] V. S. Adamchik and O. I. Marichev, "The algorithm for calculating integrals of hypergeometric type functions and its realization in REDUCE system," in Proc. International Conference on Symbolic and Algebraic Computation, Tokyo, Japan, 1990, pp. 212-224. 